\title{
Can this be considered Fondaparinux-Induced Thrombocytopenia?
}

\author{
Barbara Mariotti, Paolo Rossi, Enrico Barboni
}

Internal Medicine Department, Azienda Ospedaliero-Universitaria S.Maria della Misericordia, Udine, Italy

\begin{abstract}
We present a case of an old woman with previously documented heparin-induced thrombocytopenia (HIT), treated with fondaparinux, who presented with thrombocytopenia and venous thrombosis after exposure to a preventive dose of fondaparinux during orthopaedic surgery. Any accidental exposure to heparin was avoided. Other causes of thrombocytopenia were excluded and antigenic tests combined with clinical probability made a diagnosis of HIT likely.

Can this be considered a possible case of fondaparinux-related HIT, despite the intense and early decrease in platelets, as usually happens in rapid-onset HIT, and the fact that previous exposure to fondaparinux had occurred 5 months previously?
\end{abstract}

Keywords: Thrombosis, thrombocitopenia

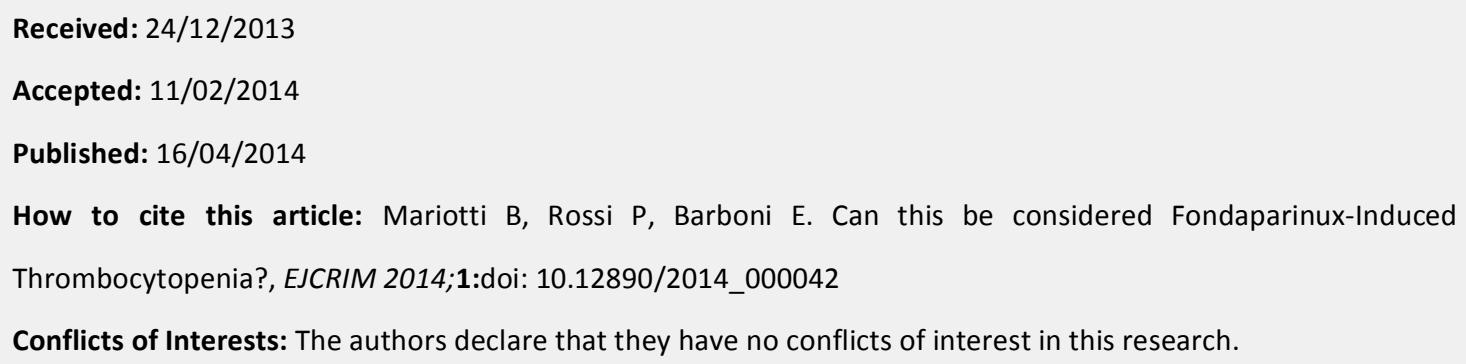

\section{Introduction}

Heparin-induced thrombocytopenia (HIT) is an immune-mediated disorder caused by antibodies against platelet factor PF4/heparin complexes characterized by an increased risk of thromboembolic complications ${ }^{1}$.

The risk is greater with the use of unfractionated heparin (UFH), ten times lower with the use of low-molecular-weight heparin (LMWH) and negligible with the use of fondaparinux, a direct $\mathrm{Xa}$ inhibitor ${ }^{2}$.

In the literature, a causal association between fondaparinux use and HIT has not been established 
and few anecdotal cases are described.

\section{Case}

We report on a case of suspected heparin-induced thrombocytopenia with thrombosis (HITT) associated with the use of fondaparinux in an 82-year-old woman with a previous HITT with documented deep venous thrombosis (DVT) and right pulmonary embolism (PE) during prophylaxis with LMWH after a minor hip fracture. The diagnosis was performed by association of high probability of HIT according to the "4Ts Score" ${ }^{1}$ and high-titre anti-PF4/heparin antibodies determined with ELISA standard (GTI-PF4), after exclusion of other causes of thrombocytopenia. She was treated with fondaparinux and, when the platelet count was normalized, with warfarin.

Five months later, still in anticoagulation with warfarin, she suffered a left hip fracture which required prosthetic hip replacement. Surgery was delayed for 5 days because of the onset of fever of unclear origin treated with a high-spectrum antibiotic.

The day of surgery, fondaparinux $2.5 \mathrm{mg} /$ day was started. The platelet count was $390,000 / \mathrm{mmc}$ and the International Normalized Ratio (INR) 2.69 before substitution with fondaparinux, such that vitamin $\mathrm{K}$ ( $2 \mathrm{mg}$ orally) was given. Because of the previous history of HITT, any incidental exposure, either intra- or peri-operatively, to UFH or LMWH was carefully avoided.

The platelet count began to fall 24 hours later, reaching a nadir on Day $3(9000 / \mathrm{mmc}$ ) (see Fig. 1).

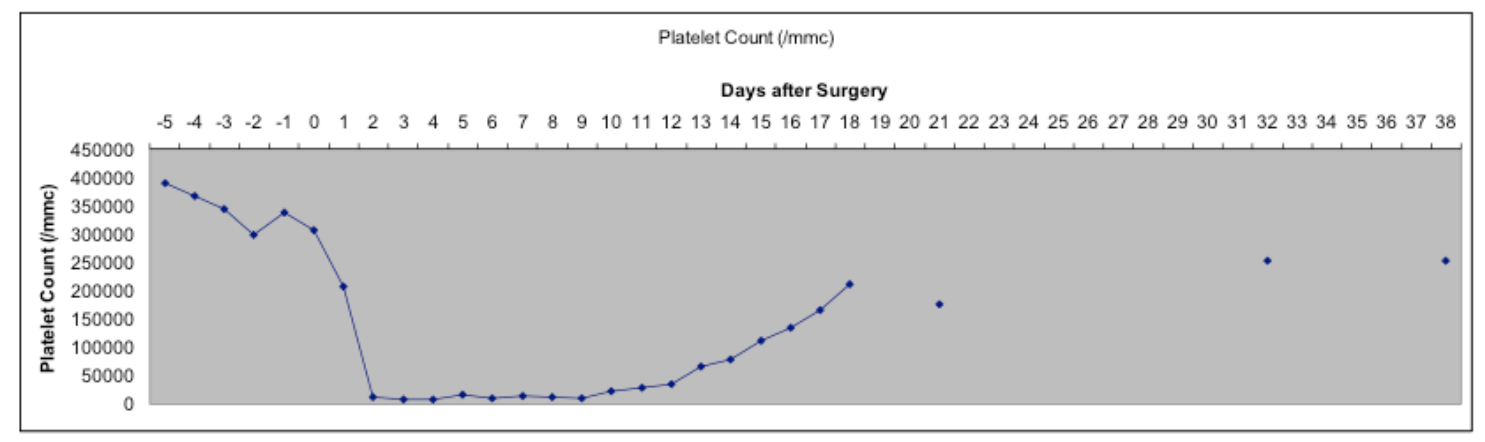
Day 0
ORTHOPEDIC SURGERY
Beginning of fondaparinux $2.5 \mathrm{mg}$
Day -3 Vitamin K administration
Day 3 Platelet count nadir $(9000 / \mathrm{mmc})$
Day 3 Beginning of lepirudine
Day 19 Re-start of warfarin
Day 26 Stop lepirudine

Figure 1: Platelets count after the beginning of fondaparinux

A bilateral PE and an extension of the previous DVT were diagnosed.

Fibrinogen, Prothrombin Time (PT), Partial Thromboplastin time (PTT) and Antithrombin III (ATIII) had normal values. Anti-heparin/PF4 antibodies (GTI-PF4) were detected in high titres (OD>1.2). The probability of HITT was intermediate (score 4-5) according to the "4T's score" and high (score 9) 
according the "HEP score"1,3. The timing and the degree of thrombocytopenia and the onset of new thrombotic manifestations in the absence of an alternative diagnosis made us suspect a relapse of HIT, although the previous exposure to fondaparinux was almost 150 days before.

Consequently, lepirudin treatment was instituted.

No cases of HIT associated with the use of fondaparinux were found in trials conducted in different clinical settings, which had been used successfully to treat $\mathrm{HIT}^{1}$.

Theoretically, the limited interactions of fondaparinux with PF4 and the lack of in vitro cross-reactivity with HIT antibodies have led to the general agreement that fondaparinux is likely non-immunogenic, suggesting its use in HIT. However, antiPF4-heparin antibodies have been detected in patients receiving thrombophylaxis with fondaparinux with an incidence similar to that observed in patients receiving LMWH enoxaparin, although without any associated HIT $^{2}$.

Warkentin hypothesized a low - though not zero - risk either of inducing HIT ex novo or of HIT relapse when antibodies are already present ${ }^{2}$. In the literature, a causal association between fondaparinux therapy and the occurrence of HIT is described only in anecdotal case reports ${ }^{1}$.

In our case, the delayed surgery, the administration of vitamin $\mathrm{K}$ and the inflammatory state may have aided the pro-thrombotic state, but no other causes of thrombocytopenia were identified. In particular, there were no signs of disseminated intravascular coagulation (DIC) or drug-induced thrombocytopenia. Can this be considered a rare case of fondaparinux-induced thrombocytopenia? Due to the rapidity and intensity of the fall in platelet count together with the severity of the clinical conditions, the present case seems to be one of "rapid-onset HIT", even though the exposure to fondaparinux was over 150 days prior and the persistence of antibodies should be considered highly unlikely. Alternatively, we could be dealing with a "spontaneous HIT" in which both clinical and serological aspects are identical to HIT, but with no heparin exposure. In this case, PF4 tetramers may have had a role in platelet activation. They may have been rendered immunogenic, through exposure to either a polyanionic bacterial surface or chondroitin sulphates released during orthopaedic surgery, or through some other, unknown, mechanism ${ }^{4}$. A delayed-onset HIT, as described by Alsaleh et al. ${ }^{5}$, triggered by the previous administration of LMWH seems less likely as five months had elapsed from the last administration of this drug, and the antibody immune response is usually short lived.

Unfortunately, we could not perform a functional assay, which would have helped to identify the nature of this event. 


\section{European Journal}

of Case Reports in

Internal Medicine

\section{Learning Points}

- Heparin-induced thromboctitopenia (HIT) is an extremely rare condition.

- A causal association between fondaparinux use and HIT has not been established and only few anecdotal cases are described

- In this report we describe a case of HIT after exposure to Fondaparinux; it is not clear if it was the drug itself causing the syndrome or if other unknown mechanisms played a role in platelet's activation.

\section{References}

1. Linkins L-A, Dans AL, Moores LK et al. Treatment and prevention of heparin-induced thrombocytopenia: antithrombotic therapy and prevention of thrombosis, 9th ed: American College of Chest Physicians Evidence-Based Clinical Practice Guidelines, Chest 2012;141;S495-S528.

2. Warkentin TE. HIT lights: a career perspective on heparin-induced thrombocytopenia, Am J Hematol 2012;87:S92-S99.

3. Cuker A, Arepally G, Crowther MA et al. The HIT Expert Probability (HEP) Score: a novel pre-test probability model for heparin-induced thrombocytopenia based on broad expert opinion, J Thromb Haemost 2010;8:2642-2650.

4. Warkentin TE. HIT paradigms and paradoxes, J Thromb Haemost 2011;9: 105-117.

5. Alsaleh KA, Al-Nasser SM, Bates SM et al. Delayed-onset HIT caused by low-molecular-weight heparin manifesting during fondaparinux propylaxis, Am J Hematol 2008;83:876-878. 